\title{
LIE INCIDENCE SYSTEMS FROM PROJECTIVE VARIETIES
}

\author{
ARJEH M. COHEN AND BRUCE N. COOPERSTEIN
}

(Communicated by Ronald M. Solomon)

\begin{abstract}
The homogeneous space $G / P_{\lambda}$, where $G$ is a simple algebraic group and $P_{\lambda}$ a parabolic subgroup corresponding to a fundamental weight $\lambda$ (with respect to a fixed Borel subgroup $B$ of $G$ in $P_{\lambda}$ ), is known in at least two settings. On the one hand, it is a projective variety, embedded in the projective space corresponding to the representation with highest weight $\lambda$. On the other hand, in synthetic geometry, $G / P_{\lambda}$ is furnished with certain subsets, called lines, of the form $g B\langle r\rangle P_{\lambda} / P_{\lambda}$ where $r$ is a preimage in $G$ of the fundamental reflection corresponding to $\lambda$ and $g \in G$. The result is called the Lie incidence structure on $G / P_{\lambda}$. The lines are projective lines in the projective embedding. In this paper we investigate to what extent the projective variety data determines the Lie incidence structure.
\end{abstract}

\section{INTRODUCTION}

Let $k$ be a field, $n$ a natural number, and let $X$ be any set of points in the projective space $\mathbf{P}\left(k^{n}\right)$. The points and lines lying on $X$ give rise to an incidence system which we shall denote by $\Delta(X)$. We are interested in the structure of these incidence systems. The main motivating examples stem from the geometries of Lie type. In these cases, the set $X$ is actually a projective variety, given as an intersection of quadrics.

1.1. Theorem (cf. [2], [8]). Suppose $G$ is a connected split semisimple algebraic group with Tits system $(B, N, W, R)$, and set $T=B \cap N$. Let $\lambda$ be a dominant weight with respect to $B$, and let $P_{\lambda}=B W_{\lambda} B$ be the corresponding parabolic subgroup of $G$. Then the $G$-module $S^{2} V(\lambda)$ contains the highest weight module $V(2 \lambda)^{*}$ with multiplicity 1 and with a $G$-invariant complement $M$. The highest weight orbit $G\left\langle v_{\lambda}\right\rangle$ of $G$ in $\mathbf{P}(V(\lambda))$ is a projective variety isomorphic to $G / P_{\lambda}$. It is the zero set of any basis of $M$ (which are homogeneous quadratic polynomials on $V(\lambda)$ ).

This result is probably well known. A proof for $k$ of characteristic 0 can be found in [2], and a more general proof in [8].

In the setting of the above theorem, let $\lambda$ be a fundamental node, say $\lambda=\omega_{j}$, and denote the corresponding reflection by $r$. Then, according to the theorem, the point set of each Lie incidence system can be identified with the zero set of all (quadratic) polynomials in $M$. Here, we recall from [3], the Lie incidence system associated with $G$ and $r$ is a pair $\Gamma=(\mathcal{P}, \mathcal{L})$, where $\mathcal{P}=G / P_{\lambda}$ and $\mathcal{L}$ consists of all

Received by the editors July 6, 1996 and, in revised form, December 18, 1996.

1991 Mathematics Subject Classification. Primary 51B25; Secondary 14L17, 14M15.

Key words and phrases. Groups of Lie type, Lie incidence systems, geometry, quadrics.

(C)1998 American Mathematical Society 


\begin{tabular}{|c|c|c|}
\hline \multicolumn{3}{|c|}{ Table 1. Geometries $Y_{n, j}$ distinct from $\Delta(\mathcal{P})$} \\
\hline$Y_{n, j}$ & $\Delta(\mathcal{P})$ & restriction \\
\hline$B_{n, n}$ & $D_{n+1, n+1}$ & \\
$C_{n, j}$ & subspace of $A_{2 n-1, j}$ & $j<n$ \\
$F_{4,3}$ & subspace of $E_{6,3}$ & \\
$F_{4,4}$ & subspace of $E_{6,1}$ & \\
$G_{2,1}$ & $B_{3,1}$ & \\
\hline
\end{tabular}

$G$-images of the 'standard' line $\left\{b\langle r\rangle P_{\lambda} \mid b \in B\right\}$. If $G$ is simple and has Lie type $Y_{n}$, we say that $\Gamma$ is the incidence system of type $Y_{n, j}$. We shall use Bourbaki's ([1]) labeling of the fundamental roots throughout.

It is well known and easily verified (see Proposition 2.1 below) that the lines of $\Gamma$ are also lines of $\Delta(\mathcal{P})$. But the converse is not always true. Counterexamples are symplectic spaces $C_{n, 1}$ in $\mathbf{P}\left(k^{2 n}\right)$ and $G_{2,1}$ in $\mathbf{P}\left(k^{7}\right)$, in which cases the lines of the corresponding $\Delta$ space are the lines of $\mathbf{P}\left(k^{2 n}\right)$, the lines of the $B_{3,1}$ quadric, respectively. In this paper, we completely classify the counterexamples.

Main Theorem. Let $G$ be a connected simple split algebraic group and choose a Tits system $(B, N, W, R)$ in $G$. Let $\lambda=\omega_{j}$ be the $j$-th fundamental weight, and let $P_{\lambda}=B W_{\lambda} B$ be the corresponding parabolic subgroup of $G$. View the variety $\mathcal{P}=G / P_{\lambda}$ as a subvariety of $\mathbf{P}\left(V(\lambda)\right.$ ) (via the map $x P_{\lambda} \mapsto k x v_{\lambda}$, where $v_{\lambda}$ is a high weight vector of $V_{\lambda}$ ), and let $\Gamma$ be the Lie incidence system of type $Y_{n, j}$. Then $\Gamma$ and $\Delta(\mathcal{P})$ have the same point set, and the lines of $\Gamma$ are also lines of $\Delta$ (that is, the Lie incidence system is embedded in $\mathbf{P}(V(\lambda)))$. Suppose $\Delta(\mathcal{P})$ and $\Gamma$ do not coincide. Then $Y_{n, j}$ is as in Table 1.

In these cases, a good description of $\Delta(\mathcal{P})$ is available, see $\S 3.4$. Also, we have a criterion, without case distinction, which tells us when two points of $\Gamma$ are on a line of $\Delta(\mathcal{P})$. It uses the Weyl group element expressing the relation between two points in $G / P_{\lambda}$ in the Bruhat decomposition of $P_{\lambda} \backslash G / P_{\lambda}$, see Proposition 2.1.

The fact that $\mathcal{P}$ is an intersection of quadrics implies that $\Delta(\mathcal{P})$ is a Gamma space, i.e., for every point $p$ and line $\ell$ either at most one or all points of $\ell$ are collinear with $p$. Also, it allows us to conclude that a projective line is a line of $\Delta(\mathcal{P})$ whenever three of its points belong to $\mathcal{P}$.

\section{Collinearity in $\Delta(\mathcal{P})$}

We first give a criterion for two points of $\Gamma$ to be collinear in $\Delta(\mathcal{P})$. We retain the notation of the Main Theorem. We shall often drop the argument $(\mathcal{P})$ and just write $\Delta$. We shall write $D^{j, j}$ to denote the set of distinguished $\left(W_{\lambda}, W_{\lambda}\right)$ coset representatives, that is, $\left\{w \in W \mid \forall_{s \in R \backslash\{r\}} l(w s)=l(s w)>l(w)\right\}$ (cf. [1]). For $w \in W$, two points $g P_{\lambda}, h P_{\lambda} \in G / P_{\lambda}$ are said to be in Bruhat relation $w$ if $g^{-1} h \in P_{\lambda} w P_{\lambda}$. Clearly, $w$ may be taken to lie in $D^{j, j}$.

2.1. Proposition. Let $p, q$ be points of $\Delta$ and suppose their Bruhat relation is $w \in D^{j, j}$. Then $p$ and $q$ are collinear in $\Delta$ if and only if $w$ is a reflection with positive root $\alpha$ such that $\langle\lambda, \alpha\rangle=1$. 
Here, $\langle\lambda, \alpha\rangle=2(\lambda, \alpha) /(\alpha, \alpha)$ for a root $\alpha$ and weight $\lambda$, and $(\cdot, \cdot)$ is the usual Euclidean inner product of the root space. The proof relies on the following

2.2. Lemma. If $p$ and $q$ are distinct collinear points of $\Delta$, then there is a maximal torus of $G$ which stabilizes $p q$, fixes both $p$ and $q$ and is transitive on the remainder of $p q$.

Proof. Without loss of generality, we may (and shall) take $p=\left\langle v_{\lambda}\right\rangle$ and $q=\left\langle v_{w \lambda}\right\rangle$, with $w$ a distinguished double $\left(W_{\lambda}, W_{\lambda}\right)$-coset representative. Here, $v_{\lambda}$ and $v_{w \lambda}$ denote weight vectors of the indicated weights. Then the standard maximal torus $T=B \cap N$ stabilizes the line $p q$ and acts as required, as $(\lambda, \alpha) \neq\left(\lambda, w^{-1} \alpha\right)$ for at least one root vector $\alpha$.

We call a subgroup of $G$ fundamental if it is a semisimple closed subgroup of $G$ normalized by a maximal torus of $G$.

2.3. Corollary. If $\ell$ is a $\Delta$ line, then there is a fundamental subgroup $S$ stabilizing $\ell$ whose induced group on $\ell$ coincides with $P S L(\ell)$. Moreover, the $\left(W_{\lambda}, W_{\lambda}\right)$-coset expressing the Bruhat relation between two distinct collinear points of $\Delta$ contains a reflection.

Proof. Let $H$ be the full stabilizer in $G$ of $\ell$. Let $T$ be a maximal torus stabilizing $\ell$ as in the proof of Lemma 2.2 (so, after conjugation, we may assume $T$ to be in $B$ ), and let $T^{\prime}$ be the connected component of the identity in the kernel of the $T$-action on $\ell$. Then $T^{\prime}$ is a torus of codimension 1 in $T$, which fixes infinitely many points of $G / P_{\lambda}$ and hence of $G / B$. So $T^{\prime}$ is the kernel of a uniquely defined positive root $\alpha$ (cf. Proposition 24.3 of [6]). Let $S$ be the commutator of the centralizer $C_{G}\left(T^{\prime}\right)$ of $T^{\prime}$ in $G$. Since $C_{G}\left(T^{\prime}\right)$ contains $T$ and is a connected reductive group of semisimple rank one (cf. Theorem 22.3 and Corollary $26.2 \mathrm{~A}$ of [6]), $S$ is a fundamental subgroup of $G$ isomorphic to $(P) S L_{2}$ and $C_{G}\left(T^{\prime}\right)=T S=T^{\prime} S$.

By a proof similar to (or using) Theorem 22.4 of [6], each component of the subvariety of $T^{\prime}$-fixed points in $G / P_{\lambda}$ is a $C_{G}\left(T^{\prime}\right)$-orbit. Since $T$ fixes two points of $\ell$ and $\ell$ is wholly contained in a single $C_{G}\left(T^{\prime}\right)$-orbit, it follows that $\ell$ is an $S$-orbit, and that $S$ acts on $\ell \cong \mathbf{P}^{1}$ in the natural way. Moreover, the non-trivial element in the Weyl group of $C_{G}\left(T^{\prime}\right)$ interchanging the two $T$-fixed points is the reflection expressing the Bruhat relation between two distinct points of $\ell$.

2.4. Proof of Proposition 2.1. Let $p=k v_{\lambda}$ and $q=k v_{w \lambda}$ be points in Bruhat relation $w$. The group $S$ of Corollary 2.3 has a 2-dimensional representation on $k v_{\lambda}+k v_{w \lambda}$, with fundamental high weight for $S$. By Corollary 2.3, $w$ is a reflection in a root, say $\alpha$, and $S$ is the fundamental subgroup isomorphic to $(P) S L_{2}$ and corresponding to this root. On the other hand, the high weight of the representation of $S$ on $k v_{\lambda}+k v_{w \lambda}$ is given by the restriction of $\lambda$ to the $\alpha$ torus, hence the condition $\langle\lambda, \alpha\rangle=1$.

Now for the converse, if $w$ is a reflection with root $\alpha$ such that $\langle\lambda, \alpha\rangle=1$, the fundamental subgroup of $G$ isomorphic to $S L_{2}$ or $P S L_{2}$ and corresponding to $\alpha$ will have a 2-dimensional invariant subspace containing $v_{\lambda}$, and, by its action on 1-spaces, will permute all the points of this projective line; hence the 2 -space will constitute a line of $\Delta$.

We shall denote by $s_{i}$ the $i$-th fundamental reflection of $W$, and, for $w \in W$, by $l(w)$ the minimal length of $r$ as a product of these fundamental reflections. If $\alpha$ is a 
root, we shall write $r_{\alpha}$ for the corresponding reflection. Thus, $s_{i}=r_{\alpha_{i}}$ and $r=s_{j}$. The following proposition provides a quick way of deciding whether a double coset contains a reflection.

2.5. Proposition. Suppose $w \in D^{j, j}$ is such that $W_{\lambda} w W_{\lambda}$ contains a reflection. Then either $w=1$ or $w$ is a reflection.

Proof. Amongst all reflections in $W_{\lambda} w W_{\lambda}$ let $r=r_{\alpha}$ be chosen with $l(r)$ minimal. Without loss of generality we may take $\alpha$ to be a positive root (otherwise replace $\alpha$ by $-\alpha$ ). We prove that either $r \in W_{\lambda}$, in which case $w=1$, or $r \in D^{j, j}$, in which case $r=w$ and hence $w$ is a reflection.

Since $r$ is a reflection it has order two, so in order to show that $r \in D^{j, j}$ it suffices to show that $r$ is a $W_{\lambda}$-distinguished coset representative; this amounts to showing that for all $i \neq j$ we have $r\left(\alpha_{i}\right)>0$. Suppose to the contrary that $r\left(\alpha_{i}\right)<0$ for some $i \neq j$. If $r\left(\alpha_{i}\right)=-\alpha_{i}$ then $r=s_{i} \in W_{\lambda}$ and consequently $w=1$. Therefore, we may assume that $r\left(\alpha_{i}\right) \neq-\alpha_{i}$. Since $r\left(\alpha_{i}\right)<0$ we have $l\left(r s_{i}\right)<l(r)$. Note that $\left(r s_{i}\right)^{-1}=s_{i} r$ since $r$ and $s_{i}$ are both reflections. This implies $l\left(r s_{i}\right)=l\left(s_{i} r\right)$. Also, $s_{i} r\left(\alpha_{i}\right)<0$ and therefore we have

$$
l\left(s_{i} r s_{i}\right)<l\left(s_{i} r\right)=l\left(r s_{i}\right)<l(r) .
$$

Since $i \neq j$, the reflection $s_{\alpha_{i}} r s_{\alpha_{i}}$ belongs to $W_{\lambda} r W_{\lambda}=W_{\lambda} w W_{\lambda}$, which contradicts the choice of $r$.

Clearly, roots in the same $W_{\lambda}$-orbit give rise to reflections in the same $\left(W_{\lambda}, W_{\lambda}\right)$ coset. Thus, it makes sense to determine all $W_{\lambda}$-orbits of roots.

2.6. Lemma. Let $\lambda=\omega_{j}$. Suppose $\mathcal{O}$ is a $W_{\lambda}$-orbit of roots $\sum_{i} a_{i} \alpha_{i}$ distinct from $W_{\lambda} \alpha_{j}$ with $a_{j}>0$ and $\langle\lambda, \alpha\rangle=1$. Then either $Y_{n, j}=C_{n, j}$ with $j<n$ and $\mathcal{O}=W_{\lambda}\left(2 \alpha_{j}+\cdots+2 \alpha_{n-1}+\alpha_{n}\right)$, or $Y_{n, j}=B_{n, n}$ and $\mathcal{O}=W_{\lambda}\left(\alpha_{n-1}+2 \alpha_{n}\right)$.

Proof. Straightforward case by case analysis. By way of example, we deal with $D_{n}$. Suppose $j<n-1$, so $\alpha_{j}=\epsilon_{j}-\epsilon_{j+1}$. Let $\alpha$ be a positive root. That is, $\alpha=\epsilon_{i} \pm \epsilon_{k}$ for some $i, k$ with $i<k$.

Suppose $i \leq j$ and $j+1 \leq k$. By transitivity of $W_{\lambda}$ on the index set $\{1, \ldots, j\}$ and (independently) on the index set $\{j+1, \ldots, n\}$, we may assume $i=j$ and $k=j+1$. Then $\alpha=\epsilon_{j}+\epsilon_{j+1}$, which is conjugate in $W_{\lambda}$ to $\epsilon_{j}-\epsilon_{j+1}$.

If $i, k \leq j$, then $\alpha=\epsilon_{i}+\epsilon_{j}$, and, by use of $W_{\lambda}$, we may assume $i=j-1$. But then $\langle\lambda, \alpha\rangle=2$.

Finally, suppose $i, k \geq j+1$. Then $i=j+1$ and, after use of $W_{\lambda}$ once more, we may assume $\alpha=\epsilon_{j+1}-\epsilon_{j+2}=\alpha_{j+1}$, contradicting $a_{j}>0$.

Next suppose $k=n$. Then any root can be transformed into $\alpha_{n-1}=\epsilon_{n-1} \pm \epsilon_{n}$ using the $A_{n}$ structure of $W_{\lambda}$. But this is in $W_{\lambda}$ so no other reflections occur.

Another restriction we can apply before classifying is:

2.7. Lemma. If $w \in W$ represents a $\Delta$ line then so does any Weyl group element less than $w$ in the Bruhat order.

Proof. Suppose $p, q \in \mathcal{P}$ are in the relation $w$. Suppose $v \in D^{j, j}$ is covered by $w$ in the Bruhat ordering restricted to $D^{j, j}$. Then there is a $\Gamma$ line $\ell$ on $q$ with a point $u$ say in relation $v$ to $p$, while all remaining points of $\ell$ are in relation $w$ to $p$. Take such a point, say $s$. The three lines $\ell, p s$, and $p q$ of the projective plane spanned by $p, q$, and $u$ all belong to $\mathcal{P}$. Consequently, all points of this plane belong to $\mathcal{P}$, 
whence $u$. But then $v$ is the Weyl group element of a $\Delta$ collinear pair of points (namely $p$ and $u$ ).

2.8. Corollary. If $\Gamma$ has $\Delta$ lines which do not belong to $\Gamma$, then there are such lines having points at $\Gamma$ distance 2.

Proof. Let $v$ and $w$ be as in the above proof. If $w$ represents a distance bigger than 2 in $\Gamma$, then replace $w$ by $v$. Continuing this way, one finds a pair of $\Delta$ collinear points at $\Gamma$ distance 2 .

\section{Proof of the Main Theorem}

3.1. Lemma. The lines of $\Gamma$ are lines of $\Delta$.

Proof. This is direct from Proposition 2.1.

3.2. Lemma. In the cases of type $E_{n, k}$ and $F_{4, j}(j \neq 3,4)$, we have $\Delta=\Gamma$.

Proof. In view of Corollary 2.8 we only need consider pairs of points at mutual distance 2 in $\Gamma$. We recall that each such pair is either symplectic (i.e., the common neighbors, together with the lines of $\Gamma$, form a Lie incidence system of type $A_{3,2}$, $D_{n, 1}$ with $n \geq 4, B_{n, 1}$, or $C_{n, 1}$ with $n \geq 2$ ) or special, in which case there is only one common neighbor.

As symplectic pairs in these cases do not correspond to $C_{n, 1}$ type incidence systems, they are not $\Delta$ collinear. But neither are special pairs, as their Bruhat relations are not reflections (this is easily checked by a look at the distinguished coset representatives and the use of Proposition 2.5, cf. [3]).

3.3. Lemma. If $\Gamma$ and $\Delta$ do not coincide, then $\Gamma$ is as in Table 1 .

Proof. Suppose $\Gamma \neq \Delta$. If $Y_{n}$ is classical, then the reflections $r_{\alpha}$ with $\langle\lambda, \alpha\rangle=$ 1 and not belonging to $W_{\lambda}\langle r\rangle W_{\lambda}$ have roots $\alpha$ as indicated in Lemma 2.6; the corresponding types $Y_{n, j}=C_{n, j}(j<n), B_{n, n}$ occur in Table 1 .

By Lemma 3.2, for the exceptional groups, we only need consider the cases $Y_{n, j}=G_{2,1}, G_{2,2}, F_{4,3}, F_{4,4}$. A direct computation with roots shows that, if $\mathcal{O}$ is a $W_{\lambda}$-orbit on roots $\alpha$ distinct from $W_{\lambda}\left(\alpha_{j}\right)$ satisfying $\langle\lambda, \alpha\rangle=1$ then we have one of the following three cases, in which $\mathcal{O}=W_{\lambda} \alpha$ :

1. $Y_{n, j}=F_{4,3}$ and $\alpha=\alpha_{2}+2 \alpha_{3}$;

2. $Y_{n, j}=F_{4,4}$ and $\alpha=\alpha_{2}+2 \alpha_{3}+2 \alpha_{4}$;

3. $Y_{n, j}=G_{2,1}$ and $\alpha=3 \alpha_{1}+\alpha_{2}$.

(The necessary computations are easily checked using LiE, cf. [7].) Due to Proposition 2.1, in these cases the spaces $\Delta$ contain indeed more lines than those of $\Gamma$.

3.4. Identification of the $\Delta$ geometries listed in Table 1. $C_{n, j}(j<n)$ : The point set of the $\Gamma$ geometry consists of all totally isotropic $j$-dimensional subspaces of a non-degenerate symplectic space $V$ of dimension $2 n$. The lines are in one-toone correspondence with the pairs $(X, Y)$ of incident totally isotropic subspaces with $\operatorname{dim}(X)=k-1$ and $\operatorname{dim}(Y)=k+1$. The associated line consists of all $k$-dimensional subspaces incident with both $X$ and $Y$. A $\Delta$-collinear but not $\Gamma$ collinear pair $\left(U_{1}, U_{2}\right)$ of totally isotropic $k$-subspaces satisfies $\operatorname{dim}\left(U_{1} \cap U_{2}\right)=k-1$ with $U_{1}+U_{2}$ not totally isotropic. The associated line consists of all the $k$-subspaces which are incident with the pair $\left(U_{1} \cap U_{2}, U_{1}+U_{2}\right)$. Consequently, this geometry is the subspace of the Grassmannian $A_{2 n-1, k}$ generated by all the totally isotropic subspaces of $V$. 
$B_{n, n}$ : The point set of $\Gamma$ consists of all $n$-dimensional totally singular subspaces of a non-degenerate orthogonal space $V$ of dimension $2 n+1$ with Witt index $n$. The $\Gamma$ lines are in one-to-one correspondence with the totally singular $n$-1-dimensional subspaces and for such a subspace $U$ the associated line consists of all the $n$ dimensional totally singular subspaces which contain it. The $\Delta$-collinear but not $\Gamma$-collinear pairs are pairs of totally singular $n$-dimensional subspaces which meet in an $n$-2-dimensional subspace. If the pair is $(X, Y)$ and $U=X \cap Y$, then the collection of all maximal singular subspaces which contain $U$, say $\mathcal{Q}(U)$, has the structure of a generalized quadrangle of type $C_{2,1}$. In this generalized quadrangle, $X, Y$ determine a "hyperbolic line" and this is the $\Delta$ line on $X$ and $Y$.

We claim that the $\Delta$ geometry is the half-spin geometry $D_{n+1, n+1}$. This can be seen as follows: Let $W$ be a $2 n+2$-dimensional orthogonal space with maximal Witt index containing $V$ as a hyperplane. This space has two classes of maximal totally singular subspaces, $\mathcal{S}_{1}, \mathcal{S}_{2}$ such that, for each totally singular subspace $Y$ of $W$ with $\operatorname{dim}(Y)=n$, there is a unique $S_{i} \in \mathcal{S}_{i}$ with $M \subset S_{i}(i=1,2)$. Let $\mathcal{S}$ be one of $\mathcal{S}_{i}(i=1,2)$. The geometry $D_{n+1, n+1}$ has as its points the set $\mathcal{S}$. Its lines are in one-to-one correspondence with the totally singular subspaces $U$ of $W$ with $\operatorname{dim}(U)=n-1$; the corresponding line consists of all the $S \in \mathcal{S}$ such that $U \subset S$.

By the above, for each maximal singular subspace $M$ in $V$ there is a unique element $S \in \mathcal{S}$ with $M \subset S$. Moreover, for $S \in \mathcal{S}$, the subspace $S \cap V$ is maximal singular in $V$. In this way we get a bijection between the points of $B_{n, n}$ and the points of $D_{n+1, n+1}$. We will denote images under this bijection by the bar convention. One can deduce from the above paragraph that $S, T \in \mathcal{S}$ are collinear if and only if $\operatorname{dim}(S \cap T)=n-1$. Moreover, the lines of $D_{n+1, n+1}$ can be described in terms of this relation (as the "double perp" of adjacent points). It therefore suffices to show that two points $X, Y$ of $B_{n, n}$ are $\Delta$-collinear if and only if $\operatorname{dim}(\bar{X} \cap \bar{Y})=$ $n-1$. Suppose first that $\operatorname{dim}(\bar{X} \cap \bar{Y})=n-1$. It then must be the case that $\operatorname{dim}(X \cap Y) \geq n-2$. If $\operatorname{dim}(X \cap Y)=n-1$ then $\{X, Y\}$ is $\Gamma$-collinear, while if $\operatorname{dim}(X \cap Y)=n-2$ then $\{X, Y\}$ is $\Delta$ but not $\Gamma$ collinear.

Conversely, suppose $\{X, Y\}$ is $\Delta$ collinear so that $\operatorname{dim}(X \cap Y) \geq n-2$. Then their images in $D_{n+1, n+1}$ satisfy $\operatorname{dim}(\bar{X} \cap \bar{Y}) \geq n-2$. Since $\bar{X}, \bar{Y} \in \mathcal{S}$, the codimension $\operatorname{dim}(\bar{X} /(\bar{X} \cap \bar{Y}))$ is even. It follows that $\operatorname{dim}(\bar{X} \cap \bar{Y})=n-1$, so $\bar{X}$ and $\bar{Y}$ are collinear points of $D_{n+1, n+1}$.

$G_{2,1}$ : The induced geometry is $B_{3,1}$. This is well known. See, for example, $\S 5.22$ of [3].

$F_{4,4}$ : The 26-dimensional module for $F_{4}$ which provides the embedding for this geometry is a hyperplane of the 27-dimensional module for $E_{6}$, which is the representation space of $E_{6,1}$. The $E_{6,1}$ points which lie in this hyperplane are the points of the $F_{4,4}$ geometry (cf. [4]). The geometry $F_{4,4}$ is an incidence system in which the geodesic closure of a symplectic pair is an incidence system of type $C_{3,1}$. Such a subsystem is called a symplecton. Two points $x, y$ in $F_{4,4}$ are $\Delta$-collinear if and only if they lie in a symplecton, in which case they are either $\Gamma$-collinear or have $\Gamma$-distance 2 and uniquely determine the symplecton $S$. The $\Delta$-line is then the hyperbolic line of $S$ which is determined by $x$ and $y$. We note that under this relation a symplecton becomes a subspace isomorphic to $\mathbf{P}\left(k^{6}\right)$. However, this is just the relation of being collinear as points of the $E_{6,1}$ geometry and therefore the $\Delta$ geometry on $F_{4,4}$ is just the subspace of the full $E_{6,1}$ geometry generated by the points in the hyperplane spanned by the points of the $F_{4,4}$ geometry. 
$F_{4,3}$ : The points of this geometry may be identified with the lines of the $F_{4,4}$ geometry. Under this identification, two lines $l_{1}, l_{2}$ are collinear in the $\Delta$ geometry when they meet and belong to a symplecton. From this we deduce that the $\Delta$ geometry on $F_{4,3}$ is the full subgeometry of $E_{6,2}$ generated by all $F_{4,4}$ lines.

3.5. Remarks. A. The Main Theorem can easily be extended to deal with nonmaximal parabolic subgroups $P_{\lambda}$, by replacing the fundamental weight $\lambda$ by sums of distinct fundamental weights. (Note that the latter condition is imperative, for if some fundamental weight occurs more than once in $\lambda$, some $\Gamma$-lines are no longer $\Delta$-lines.)

Let $I_{0}$ be the set of indices $j$ for which $\omega_{j}$ occurs in $\lambda$. Then there are $\left|I_{0}\right|$ different kinds of $\Gamma$-line, one for each root in $I_{0}$ (cf. [5]). Now suppose $\left|I_{0}\right|>1$. Set $I=\{1, \cdots, n\}$ and $J=I \backslash I_{0}$ (so that $P_{\lambda}=P_{J}$ ). Now $\Delta$-lines consisting of non- $\Gamma$ collinear points exist provided:

(i) $I$ has two root lengths; and

(ii) there is a short root $\alpha \in I_{0}$ whose connected component in $J \cup\{\alpha\}$ has a long root.

We remark that this can occur for at most one root in $I_{0}$ and consequently there is at most one class of $\Delta$-lines which are not $\Gamma$ lines. Suppose (i) and (ii) occur. Then, for some $k$, there is a subset $J^{\prime}$ of $J \cup\{\alpha\}$ with $\alpha \in J^{\prime}$ of type $C_{k}$. If we let the $C_{k}$-fundamental roots in $J^{\prime}$ be $\alpha=\beta_{1}, \beta_{2}, \ldots, \beta_{k}$, then the reflection

$$
r=r_{\beta_{1}} r_{\beta_{2}} \cdots r_{\beta_{k-1}} r_{\beta_{k}} r_{\beta_{k-1}} \cdots r_{\beta_{2}} r_{\beta_{1}}
$$

is in $D_{J, J}$ (the set of distinguished $\left(W_{J}, W_{J}\right)$-double coset representatives) and gives $\Delta$ but not $\Gamma$-collinearity.

B. In the cases of Table 1 where $\operatorname{Aut}(\Delta)=\operatorname{Aut}(\Gamma)$, the $\Gamma$-lines can be recovered from $\Delta$ in an intrinsic way. The relevant cases are $C_{n, j}(j<n), F_{4,4}$, and $F_{4,3}$. We describe this in the $C_{n, j}$ case. The arguments for $F_{4,4}$ and $F_{4,3}$ are similar. In the $\Delta$-geometry on $C_{n, j}(j<n)$, there are two types of maximal singular subspaces: for a point of the Lie incidence system, that is, a totally isotropic subspace $U$ of dimension $j-1$, we have the collection $M(U)$ of all the totally isotropic $j$ dimensional subspaces which contain $U$. As a space the subspace $M(U)$ of $\Delta$ is isomorphic to $\mathbf{P}\left(k^{2 n-2 j+2}\right)$. For a totally singular $j+1$-dimensional subspace $W$ there is the collection $N(W)$ of all hyperplanes of $W$. This is a singular subspace of $\Gamma$ isomorphic to $\mathbf{P}\left(k^{j+1}\right)$. On the other hand, $M(U)$ is a symplecton of $\Gamma$ and two distinct subspaces always intersect in a singular subspace of $\Gamma$. Consequently, the $\Gamma$-lines are those $\Delta$-lines which belong to more than one $\Delta$-maximal singular subspace.

\section{ACKNOWLEDGMENTS}

We are grateful to Michel Brion for suggesting improvements of an earlier version of this paper, in particular for the one leading to the present (nicer) proof of Corollary 2.3.

\section{REFERENCES}

1. N. Bourbaki, Groupes et algèbres de Lie, Chap. IV, V, VI, Hermann, Paris, 19 68. MR 39:1590

2. M. Brion, Représentations exceptionnelles des groupes semi-simples, Ann. Scient. Éc. Norm. Sup. 18 (1985), 345-387. MR 87e:14043 
3. A.M. Cohen, Point-line spaces associated with buildings, Handbook of Incidence Geometry, Buildings and Foundations (ed. F. Buekenhout), Elsevier, Amsterdam (ISBN 044488355 X), 1995, pp. 647-737. MR 96k:51009

4. A.M. Cohen and B.N. Cooperstein, The 2-spaces of the standard $E_{6}(q)$-module, Geometriae Dedicata 24 (1988), 467-480. MR 89c:51013

5. B.N. Cooperstein, Some geometries associated with parabolic representations of groups of Lie type, Canad. J. Math. 28 (1976), 1021-1031. MR 54:383

6. J.E. Humphreys, Linear algebraic groups, Graduate Texts in Mathematics, vol. 21, SpringerVerlag, Berlin, 1975. MR 53:633

7. M.A.A. van Leeuwen, A.M. Cohen, B. Lisser, LiE manual, describing version 2.0, CAN, Amsterdam, 1992.

8. A. Ramanathan, Equations defining Schubert varieties and Frobenius splitting of diagonals, Publ. Math. I.H.E.S. Sup. 65 (1987), 61-90. MR 88k:14032

Fac. Wisk. en Inf., TUE Postbus 513, 5600 MB Eindhoven, The Netherlands

E-mail address: amc@win.tue.nl

Department of Mathematics, University of California, Santa Cruz, California 95064

E-mail address: coop@cats.ucsc.edu 